\title{
Parton distributions in the virtual photon up to NNLO in QCD and factorization scheme dependence
}

\section{Takahiro Ueda}

High Energy Accelerator Research Organization (KEK)

1-1 Oho, Tsukuba, Ibaraki 305-0801, Japan

E-mail: uedat@post.kek.jp

\section{Tsuneo Uematsu}

Dept. of Physics, Graduate School of Science, Kyoto University

Yoshida, Kyoto 606-8501, Japan

E-mail: uematsu@scphys.kyoto-u.ac.jp

\section{Ken Sasaki*}

Dept. of Physics, Faculty of Engineering, Yokohama National University Yokohama 240-8501, Japan

E-mail: sasaki@phys.ynu.ac.jp

\begin{abstract}
Parton distributions in the virtual photon target are investigated in perturbative QCD up to the next-to-next-to-leading order (NNLO). In the case $\Lambda^{2} \ll P^{2} \ll Q^{2}$, where $-Q^{2}\left(-P^{2}\right)$ is the mass squared of the probe (target) photon, parton distributions are predicted completely up to the NNLO, but they are factorization-scheme-dependent. We analyze parton distributions in two different factorization schemes, namely $\overline{\mathrm{MS}}$ and $\mathrm{DIS}_{\gamma}$ schemes, and discuss their scheme dependence. We show that the factorization-scheme dependence is characterized by the large- $x$ behaviors of quark distributions in the virtual photon. Gluon distribution is predicted to be very small in absolute value except in the small- $x$ region.
\end{abstract}

8th International Symposium on Radiative Corrections (RADCOR)

October 1-5 2007

Florence, Italy

\footnotetext{
* Speaker.
} 


\section{Introduction}

In the previous talk (see this Proceedings), Uematsu presented our analysis on the structure functions of the virtual photon in perturbative QCD (pQCD) up to the next-to-next-to-leading order (NNLO) [1]. The structure functions $F_{2}^{\gamma}$ and $F_{L}^{\gamma}$ were studied in the kinematical region, $\Lambda^{2} \ll P^{2} \ll Q^{2}$, where $-Q^{2}\left(-P^{2}\right)$ is the mass squared of the probe (target) photon and $\Lambda$ is the QCD scale parameter. The advantage in studying the virtual photon target in this kinematical region is that we can predict the whole structure functions, their shape and magnitude, by the perturbative method in QCD. In fact, using the recent calculation results of the three-loop anomalous dimensions for the quark and gluon operators and of the three-loop photon-quark and photon-gluon splitting functions [2], we could make a definite prediction for the virtual photon structure function $F_{2}^{\gamma}\left(x, Q^{2}, P^{2}\right)$ up to the NNLO in pQCD.

In parton picture, structure functions are expressed as convolutions of the parton distributions in the target and the coefficient functions. Thus, in kinematical region $\Lambda^{2} \ll P^{2} \ll Q^{2}$, a definite prediction is also possible for the parton distribution functions in the virtual photon in pQCD. However, it is well known that parton distributions obtained from the next-to-leading order (NLO) (and also the NNLO) analyses are dependent on the factorization scheme employed. It is possible that parton distributions obtained in one factorization scheme may be more appropriate to use than those in other schemes. In this talk I report our analysis on the parton distributions in the target photon. The parton distributions of the flavor singlet and non-singlet quarks and gluon are investigated up to the NNLO in two factorization schemes, namely $\overline{\mathrm{MS}}$ and DIS $\gamma$ schemes.

\section{QCD improved parton model}

Let $q_{S}^{\gamma}\left(x, Q^{2}, P^{2}\right), q_{N S}^{\gamma}\left(x, Q^{2}, P^{2}\right), G^{\gamma}\left(x, Q^{2}, P^{2}\right), \Gamma^{\gamma}\left(x, Q^{2}, P^{2}\right)$ be the flavor singlet-, non-singletquark, gluon, and photon distribution functions, respectively, in the unpolarized virtual photon with mass $-P^{2}$. In the leading order of the electromagnetic coupling constant, $\alpha=e^{2} / 4 \pi, \Gamma^{\gamma}$ does not evolve with $Q^{2}$ and is set to be $\Gamma^{\gamma}\left(x, Q^{2}, P^{2}\right)=\delta(1-x)$. Introducing a row vector $\boldsymbol{q}^{\gamma}=\left(q_{S}^{\gamma}, G^{\gamma}, q_{N S}^{\gamma}\right)$, the parton distribution functions in the virtual photon satisfy the DGLAP evolution equations which, in space of the Mellin moments, are given in a compact matrix form as

$$
\frac{d \boldsymbol{q}^{\gamma}\left(n, Q^{2}, P^{2}\right)}{d \ln Q^{2}}=\boldsymbol{k}\left(n, Q^{2}\right)+\boldsymbol{q}^{\gamma}\left(n, Q^{2}, P^{2}\right) P\left(n, Q^{2}\right)
$$

where the row vector $\boldsymbol{k}\left(n, Q^{2}\right)=\left(k_{S}, k_{G}, k_{N S}\right)$ represents the splitting functions from photon into quark and gluon, while the $3 \times 3$ matrix $P\left(n, Q^{2}\right)$ expresses the splitting functions of quark and gluon. Henceforth we omit the obvious $n$-dependence for simplicity.

We expand the splitting functions $\boldsymbol{k}\left(Q^{2}\right)$ and $P\left(Q^{2}\right)$ in powers of the QCD coupling $\alpha_{S}\left(Q^{2}\right)$ as

$$
\begin{aligned}
\boldsymbol{k}\left(Q^{2}\right) & =\frac{\alpha}{2 \pi} \boldsymbol{k}^{(0)}+\frac{\alpha \alpha_{s}\left(Q^{2}\right)}{(2 \pi)^{2}} \boldsymbol{k}^{(1)}+\frac{\alpha}{2 \pi}\left[\frac{\alpha_{s}\left(Q^{2}\right)}{2 \pi}\right]^{2} \boldsymbol{k}^{(2)}+\cdots \\
P\left(Q^{2}\right) & =\frac{\alpha_{s}\left(Q^{2}\right)}{2 \pi} P^{(0)}+\left[\frac{\alpha_{s}\left(Q^{2}\right)}{2 \pi}\right]^{2} P^{(1)}+\left[\frac{\alpha_{s}\left(Q^{2}\right)}{2 \pi}\right]^{3} P^{(2)}+\cdots
\end{aligned}
$$


Then, introducing $t$ instead of $Q^{2}$ as the evolution variable [3], $t \equiv \frac{2}{\beta_{0}} \ln \frac{\alpha_{s}\left(P^{2}\right)}{\alpha_{s}\left(Q^{2}\right)}$, we find that $\boldsymbol{q}^{\gamma}(t)(=$ $\left.\boldsymbol{q}^{\gamma}\left(n, Q^{2}, P^{2}\right)\right)$ satisfies the following inhomogenious differential equation:

$$
\begin{aligned}
\frac{d \boldsymbol{q}^{\gamma}(t)}{d t}= & \frac{\alpha}{2 \pi}\left\{\frac{2 \pi}{\alpha_{s}} \boldsymbol{k}^{(0)}+\left[\boldsymbol{k}^{(1)}-\frac{\beta_{1}}{2 \beta_{0}} \boldsymbol{k}^{(0)}\right]+\frac{\alpha_{s}}{2 \pi}\left[\boldsymbol{k}^{(2)}-\frac{\beta_{1}}{2 \beta_{0}} \boldsymbol{k}^{(1)}+\frac{1}{4}\left(\left(\frac{\beta_{1}}{\beta_{0}}\right)^{2}-\frac{\beta_{2}}{\beta_{0}}\right) \boldsymbol{k}^{(0)}\right]+\mathscr{O}\left(\alpha_{s}^{2}\right)\right\} \\
+ & \boldsymbol{q}^{\gamma}(t)\left\{P^{(0)}+\frac{\alpha_{s}}{2 \pi}\left[P^{(1)}-\frac{\beta_{1}}{2 \beta_{0}} P^{(0)}\right]\right. \\
& \left.+\frac{\alpha_{s}^{2}}{(2 \pi)^{2}}\left[P^{(2)}-\frac{\beta_{1}}{2 \beta_{0}} P^{(1)}+\frac{1}{4}\left(\left(\frac{\beta_{1}}{\beta_{0}}\right)^{2}-\frac{\beta_{2}}{\beta_{0}}\right) P^{(0)}\right]+\mathscr{O}\left(\alpha_{s}^{3}\right)\right\}, \quad(2.4)
\end{aligned}
$$

where we have used the fact that $\alpha_{s}\left(Q^{2}\right)$ satisfies $\frac{d \alpha_{s}\left(Q^{2}\right)}{d \ln Q^{2}}=-\beta_{0} \frac{\alpha_{s}\left(Q^{2}\right)^{2}}{4 \pi}-\beta_{1} \frac{\alpha_{s}\left(Q^{2}\right)^{3}}{(4 \pi)^{2}}-\beta_{2} \frac{\alpha_{s}\left(Q^{2}\right)^{4}}{(4 \pi)^{3}}$. We look for the solution $\boldsymbol{q}^{\gamma}(t)$ in the following form:

$$
\boldsymbol{q}^{\gamma}(t)=\boldsymbol{q}^{\gamma(0)}(t)+\boldsymbol{q}^{\gamma(1)}(t)+\boldsymbol{q}^{\gamma(2)}(t)
$$

where the first, second and third terms represent the solution in the LO, NLO and NNLO. The initial conditions for $\boldsymbol{q}^{\gamma(0)}, \boldsymbol{q}^{\gamma(1)}$ and $\boldsymbol{q}^{\gamma(2)}$ are obtained as follows: For $-p^{2}=P^{2} \gg \Lambda^{2}$, the photon matrix elements of the hadronic operators $O_{n}^{i}(i=S, G, N S$, corresponding to the parton distributions, $q_{S}^{\gamma}, G^{\gamma}, q_{N S}^{\gamma}$, respectively) can be calculated perturbatively. Renormalizing at $\mu^{2}=P^{2}$, we obtain at the two-loop level

$$
\left.\left\langle\gamma(p)\left|O_{n}^{i}(\mu)\right| \gamma(p)\right\rangle\right|_{\mu^{2}=P^{2}}=\frac{\alpha}{4 \pi}\left\{A_{n}^{i(1)}+\frac{\alpha_{s}\left(P^{2}\right)}{4 \pi} A_{n}^{i(2)}\right\}, \quad i=S, G, N S .
$$

The $A_{n}^{i(1)}$ and $A_{n}^{i(2)}$ terms represent the operator mixing between the hadronic operators and photon operators in the NLO and NNLO, respectively, and the operator mixing implies that there exist parton distributions in the photon. Thus we have, at $\mu^{2}=P^{2}$ (or at $t=0$ ),

$$
\boldsymbol{q}^{\gamma(0)}(0)=0, \quad \boldsymbol{q}^{\gamma(1)}(0)=\frac{\alpha}{4 \pi} \boldsymbol{A}_{n}^{(1)}, \quad \boldsymbol{q}^{\gamma(2)}(0)=\frac{\alpha \alpha_{s}\left(P^{2}\right)}{(4 \pi)^{2}} \boldsymbol{A}_{n}^{(2)},
$$

where $\boldsymbol{A}_{n}^{(l)}=\left(A_{n}^{S(l)}, A_{n}^{G(l)}, A_{n}^{N S(l)}\right)$ with $l=1,2$. Actually, quark distributions emerge, at $\mu^{2}=P^{2}$, in the order $\alpha$ and gluon distribution first appears in the order $\alpha \alpha_{s}$.

With these initial conditions (2.7), we solve Eq.(2.4). The solution, which are expressed in terms of $\boldsymbol{q}^{\gamma(0)}(t), \boldsymbol{q}^{\gamma(1)}(t)$ and $\boldsymbol{q}^{\gamma(2)}(t)$, will be reported elsewhere [4].

\section{Parton distributions in $\overline{\mathrm{MS}}$ and $\mathrm{DIS}_{\gamma}$ schemes}

The structure functions are expressed as convolutions of the parton distributions and the coefficient functions. Thus the Mellin moments of the structure function $F_{2}^{\gamma}$ are written as

$$
F_{2}^{\gamma}\left(n, Q^{2}, P^{2}\right)=\tilde{\boldsymbol{q}}^{\gamma}\left(n, Q^{2}, P^{2}\right) \cdot \tilde{\boldsymbol{C}}^{\gamma}\left(n, Q^{2}\right),
$$

where $\widetilde{\boldsymbol{q}}^{\gamma}=\left(q_{S}^{\gamma}, G^{\gamma}, q_{N S}^{\gamma}, \Gamma^{\gamma}\right)$ and $\widetilde{\boldsymbol{C}}^{\gamma}=\left(C_{2}^{S}, C_{2}^{G}, C_{2}^{N S}, C_{2}^{\gamma}\right)$. Although $F_{2}^{\gamma}$ is a physical quantity and thus is unique, there remains a freedom in the factorization of $F_{2}^{\gamma}$ into $\widetilde{\boldsymbol{q}}^{\gamma}$ and $\widetilde{\boldsymbol{C}}^{\gamma}$. Given the formula (3.1), we can always redefine $\widetilde{\boldsymbol{q}}^{\gamma}$ and $\widetilde{\boldsymbol{C}}^{\gamma}$ as follows:

$$
\left.\widetilde{\boldsymbol{q}}^{\gamma}\left(n, Q^{2}, P^{2}\right)\right|_{a} \equiv \widetilde{\boldsymbol{q}}^{\gamma}\left(n, Q^{2}, P^{2}\right) Z_{a}\left(n, Q^{2}\right),\left.\quad \tilde{\boldsymbol{C}}^{\gamma}\left(n, Q^{2}\right)\right|_{a} \equiv Z_{a}\left(n, Q^{2}\right)^{-1} \tilde{\boldsymbol{C}}^{\gamma}\left(n, Q^{2}\right),
$$




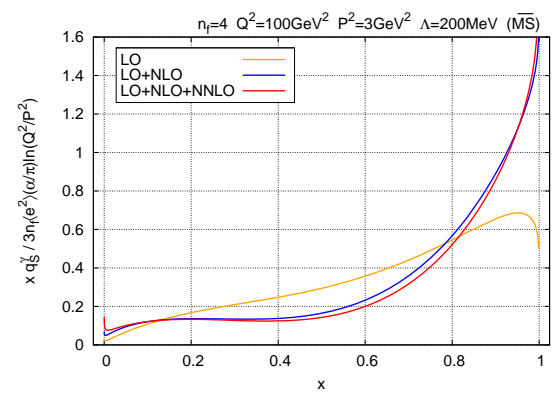

(a)

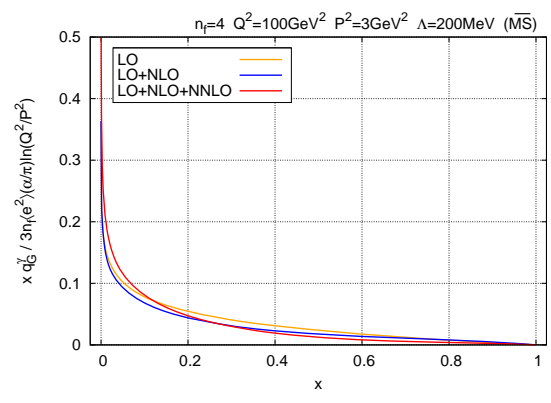

(b)

Figure 1: Parton distributions in the photon in $\overline{\mathrm{MS}}$ scheme for $n_{f}=4, Q^{2}=100 \mathrm{GeV}^{2}, P^{2}=3 \mathrm{GeV}^{2}$ with $\Lambda=200 \mathrm{MeV}$ : (a) Singlet-quark and (b) gluon

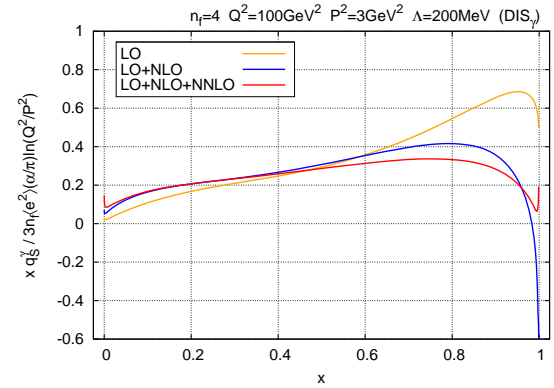

(a)

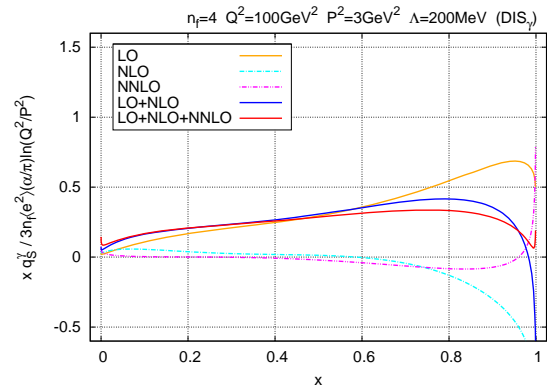

(b)

Figure 2: (a) Singlet quark distribution in DIS $\gamma$ scheme for $n_{f}=4, Q^{2}=100 \mathrm{GeV}^{2}, P^{2}=3 \mathrm{GeV}^{2}$ with $\Lambda=200 \mathrm{MeV}$. (b) LO, NLO, and NNLO contributions.

where $\left.\widetilde{\boldsymbol{q}}^{\gamma}\left(n, Q^{2}, P^{2}\right)\right|_{a}$ and $\left.\widetilde{\boldsymbol{C}}^{\gamma}\left(n, Q^{2}\right)\right|_{a}$ correspond to the quantities in a new factorization scheme- $a$. We have studied the parton distributions in two factorization schemes, $\overline{\mathrm{MS}}$ and $\mathrm{DIS}_{\gamma}$ schemes.

\section{(i) The $\overline{\mathrm{MS}}$ scheme}

This is the only scheme in which the quantities $\boldsymbol{k}^{(1)}, \boldsymbol{k}^{(2)}, P^{(1)}, P^{(2)}, \beta_{1}$ and $\beta_{2}$ appearing in Eq.(2.4) were actually calculated. When these quantities are used for the solution of Eq.(2.4), we obtain the parton distributions in $\overline{\mathrm{MS}}$ scheme. In Fig.1 we plot (a) the singlet quark distribution $x q_{S}^{\gamma}\left(x, Q^{2}, P^{2}\right)$ and (b) the gluon distribution $x G^{\gamma}\left(x, Q^{2}, P^{2}\right)$ in units of $\left(3 n_{f}\left\langle e^{2}\right\rangle \alpha / \pi\right) \ln \left(Q^{2} / P^{2}\right)$ for the case $n_{f}=4, Q^{2}=100 \mathrm{GeV}^{2}, P^{2}=3 \mathrm{GeV}^{2}$, and $\Lambda=200 \mathrm{MeV}$. We see that both the (LO+NLO) and (LO+NLO+NNLO) curves show the similar behaviors in almost whole $x$ region, which means that the NNLO contribution is small. The behaviors of these two curves, however, are quite different from the LO curve. They lie below the LO curve for $0.2<x<0.8$, but diverge as $x \rightarrow 1$. Compared with the quark distribution, the gluon distribution $\left.x G^{\gamma}\right|_{\overline{\mathrm{MS}}}$ is very small in absolute value except in the small- $x$ region. Concerning the non-singlet quark distribution $q_{N S}\left(x, Q^{2}, P^{2}\right)$, we find that when we take into account the charge factors, such as $\left\langle e^{2}\right\rangle=\sum_{i} e_{i}^{2} / n_{f}$ and $\left\langle e^{4}\right\rangle=\sum_{i} e_{i}^{4} / n_{f}$, it falls on the singlet quark distribution in almost the whole $x$ region; namely the two "normalized" distributions $\tilde{q}_{S}^{\gamma} \equiv q_{S}^{\gamma} /\left\langle e^{2}\right\rangle$ and $\widetilde{q}_{N S}^{\gamma} \equiv q_{N S}^{\gamma} /\left(\left\langle e^{4}\right\rangle-\left\langle e^{2}\right\rangle^{2}\right)$ mostly overlap except at the very small $x$ region. This situation is the same in both $\overline{\mathrm{MS}}$ and $\mathrm{DIS}_{\gamma}$ schemes. 


\section{(ii) The DIS $\gamma$ scheme}

An interesting factorization scheme, which is called DIS $\gamma$, was introduced some time ago into the NLO analysis of the real photon structure function $F_{2}^{\gamma}\left(x, Q^{2}\right)$. Glück, Reya and Vogt [5] observed that, in the $\overline{\mathrm{MS}}$ scheme, the $\ln (1-x)$ term in the photonic coefficient function $C_{2}^{\gamma}(x)$, which becomes negative and divergent for $x \rightarrow 1$, drives $F_{2}^{\gamma}$ to negative values as $x \rightarrow 1$, leading to a strong difference between the LO and the NLO results for $F_{2}^{\gamma}$ in the large- $x$ region. They introduced the DIS $\gamma$ scheme in which the photonic coefficient function $C_{2}^{\gamma}$ is absorbed into the quark distributions. We analyzed the parton distributions of the virtual photon in the DIS $\mathrm{S}_{\gamma}$ scheme up to the NNLO. The details will be reported elsewhere [4]. In Fig.2 (a) we show the result of the singlet quark distribution $\left.x q_{S}^{\gamma}\left(x, Q^{2}, P^{2}\right)\right|_{\mathrm{DIS} \gamma}$ for $n_{f}=4, Q^{2}=100 \mathrm{GeV}^{2}, P^{2}=3 \mathrm{GeV}^{2}$, and $\Lambda=200 \mathrm{MeV}$. The three curves (LO, LO+NLO, LO+NLO+NNLO) rather overlap below $x=0.6$. But absorbing the photonic coefficient function $C_{2}^{\gamma}$ into the quark distributions in the DIS ${ }_{\gamma}$ scheme has much effect on their large- $x$ behaviors: Unlike the $\overline{\mathrm{MS}}$ scheme, the (LO+NLO) curve goes under the LO one at $x \approx 0.6$ and the difference between the two grows as $x \rightarrow 1$. Adding the NNLO contribution makes the difference bigger at large $x$ except near $x=1$. At very close to $x=1$ the (LO+NLO+NNLO) curve shows a sudden surge. In order to see details, we plot, in Fig.2(b), the NLO and NNLO contributions in the DIS ${ }_{\gamma}$ scheme. We see that the NLO contribution is large and negative for $x>0.8$, while the NNLO contribution remains to be very small until very close to $x=1$ and then blows up. The gluon distribution $\left.x G^{\gamma}\right|_{\mathrm{DIS}_{\gamma}}$ is predicted to be the same as $\left.x G^{\gamma}\right|_{\overline{\mathrm{MS}}}$ up to the NNLO.

\section{Summary}

The parton distributions in the virtual photon target are analyzed up to the NNLO in the $\overline{\mathrm{MS}}$

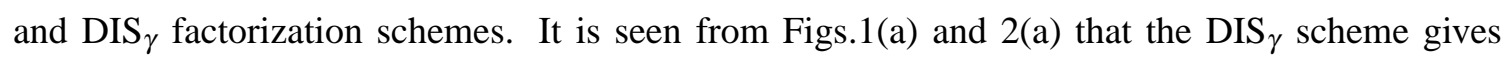
a better behavior for $x q_{S}^{\gamma}\left(x, Q^{2}, P^{2}\right)$ than $\overline{\mathrm{MS}}$ in the sense that the DIS ${ }_{\gamma}$ curve is closer to the LO result. Finally, it is noted that the (LO+NLO+NNLO) curve for $\left.x q_{S}^{\gamma}\left(x, Q^{2}, P^{2}\right)\right|_{\text {DIs }}$ shows a sudden surge at very close to $x=1$. In fact, the NNLO contribution, $\left.q_{S}^{\gamma(2)}\right|_{\mathrm{DIS}_{\gamma}}$, diverges as $\left.q_{S}^{\gamma(2)}\right|_{\text {DIS }_{\gamma}} \propto$ $\frac{8}{3}\left[-\ln ^{3}(1-x)\right]$. This may hint a necessity of considering the resummation near $x=1$.

\section{Acknowledgments}

I would like to thank the organizers of RADCOR 2007 for their hospitality at this very productive symposium.

\section{References}

[1] T. Ueda, K. Sasaki and T. Uematsu, Phys. Rev. D75 (2007) 114009.

[2] S. Moch, J.A.M. Vermaseren and A. Vogt, Nucl. Phys. B688 (2004) 101; B691 (2004) 129; Acta Phys. Pol. B37 (2006) 683 [hep-ph/0511112].

[3] W. Furmanski and R. Petronzio, Z. Phys. C11 (1982) 293.

[4] T. Ueda, K. Sasaki and T. Uematsu, in preparation.

[5] M. Glück, E. Reya and A. Vogt, Phys. Rev. D45 (1992) 3986. 